\title{
ANEMIA EM GESTANTES ATENDIDAS NA REDE PÚBLICA DE SAÚDE DE UM MUNICÍPIO DO SUL DE MINAS GERAIS
}

\author{
ANEMIA IN PREGNANT WOMEN ASSISTED IN PUBLIC HEALTH SERVICES OF CITY OF \\ THE SOUTH OF MINAS GERAIS
}

\author{
Alice Aparecida de Oliveira Luiz ${ }^{\mathrm{a}^{*}}$, Milena Bernardes Santos ${ }^{\mathrm{b}^{*}}$, Tábatta Renata Pereira de Brito ${ }^{\mathrm{c}^{*}}$, \\ Clícia Valim Côrtes Gradim ${ }^{\mathrm{d}^{*}}$, Daniela Braga Lima ${ }^{\mathrm{e}^{*}}$ \\ Alice-aparecida2011@hotmail.com a, milenabs@hotmail.com ${ }^{\mathrm{b}}$, tabatta.brito@unifal-mg.edu.br ${ }^{\mathrm{c}}$, cliciagradim@gmail.com ${ }^{\mathrm{d}}$, \\ danibraga@unifal-mg.edu.bre \\ Universidade Federal de Alfenas- UNIFAL/MG
}

\section{RESUMO}

Data de recebimento do artigo: 19/02/2018

Data de aceite do artigo: 18/02/2019

Introdução: A anemia gestacional constitui o problema nutricional em nosso meio, podendo acarretar efeitos deletérios na saúde do binômio mãe-filho. Objetivo: Verificar a ocorrência de anemia em gestantes assistidas em serviços públicos de saúde. Métodos: Estudo de corte transversal com gestantes adultas cadastradas e acompanhadas durante o pré-natal em Unidades de Saúde da Família de um município do sul de Minas Gerais. A anemia gestacional foi identificada por um nível de hemoglobina $<11 \mathrm{~g} / \mathrm{dL}$. Aplicou-se um questionário estruturado com informações socioeconômicas, obstétricas, estilo de vida, nutricionais e a dosagem dos parâmetros hematológicos. Os dados foram tratados por meio das análises descritivas e bivariadas. Resultados: A ocorrência da anemia encontrada foi de 11,43\% com maior frequência entre as gestantes na faixa etária de 31 a 40 anos, com baixa escolaridade, solteiras, com início do acompanhamento no terceiro trimestre de gestação, tabagistas e com baixo peso pré-gestacional. Observou-se consumo de dietas inadequadas em relação aos macros e micronutrientes. Conclusões: Os resultados evidenciaram baixa prevalência de anemia, caracterizando um problema leve de saúde pública e uma situação importante quanto a inadequação do consumo alimentar. Contudo, faz-se necessário novas investigações sobre os fatores associados à ocorrência dessa deficiência nutricional, destacando-se a importância da assistência à saúde como fator de prevenção à anemia e demais intercorrências gestacionais.

Palavras-chave: Anemia; gravidez; atenção primária à saúde.

\begin{abstract}
Introduction: Gestational anemia constitutes the nutritional problem in our environment, which may cause deleterious effects on the health of mother-child. Objectives: To verify the occurrence of anemia in pregnant women assisted in public health services. Methods: Cross-sectional study with adult pregnant women enrolled and accompanied during prenatal care at the Family Health of city of the south of Minas Gerais. Gestational anemia was identified as haemoglobin $<11 \mathrm{~g} / \mathrm{dL}$. The application of a structured questionnaire with socioeconomic, obstetric, lifestyle, nutritional and hematological parameters. Data were treated through descriptive and bivariate analyses. Results: The occurrence of anemia was $11.43 \%$, with higher frequencies among pregnant women in the age group of 31 to 40 years old, with low schooling, unmarried, with the onset of follow-up in the third trimester of gestation, smokers and with low pre gestational weight. Consumption of inadequate diets was observed in relation to macros and micronutrients. Conclusions: The results showed a low prevalence of anemia, characterizing a mild public health problem and an important situation regarding the inadequacy of food consumption, since it can have consequences for pregnant women and the fetus. However, new research is needed on the factors associated with the occurrence of this nutritional deficiency, highlighting the importance of health care as a factor to prevent anemia and other gestational intercurrences.
\end{abstract}

Key words: Anemia; pregnancy; primary health care. 


\section{INTRODUÇÃO}

A gestação é caracterizada por um período de intensas alterações fisiológicas, metabólicas e endócrinas ${ }^{1}$. Entre as alterações, destaca-se um aumento significativo na demanda metabólica pelo mineral ferro, em razão de uma hematopoese aumentada, para suprir tanto a necessidade da mãe quanto do feto, fato este que pode contribuir para o risco nesse grupo de aparecimento de anemia ferropriva constituindo-se, assim, em momento de maior vulnerabilidade ${ }^{2}$.

A anemia na gestação constitui-se o problema nutricional de maior prevalência, pois pode causar efeitos deletério no binômio mãe-filho. A anemia é responsável por: maior taxa de mortalidade materna e perinatal, prematuridade, baixo peso ao nascer, anemia no primeiro ano de vida do recém-nascido, entre outros ${ }^{3}$. Entre fatores que associam à anemia durante a gestação, destacam-se o baixo nível educacional e socioeconômico materno, idade gestacional mais avançada, multíparas, ausência de suplementação de ferro, ingestão alimentar inadequada e deficiente de ferro e reservas maternas inadequadas do mineral ${ }^{4,5}$.

No Brasil, não há uma pesquisa de abrangência nacional sobre a prevalência da anemia em gestantes, mas estudos pontuais indicam disparidades regionais importantes, com variação de 6,3\% no Sul a 33,5\% no Nordeste ${ }^{6,7}$. Em virtude desse cenário, a redução da anemia foi inserida entre as metas globais de nutrição para 2025, com perspectiva de declínio de $50 \%$ entre as mulheres em idade reprodutiva ${ }^{2,8}$.

Visto que o município em estudo possuiu um atendimento de pré-natal na atenção primária consolidado desde 1981 e por não haver dados sobre a prevalência da anemia no período gestacional o presente estudo teve como objetivo verificar a ocorrência da mesma em gestantes assistidas nos serviços públicos de saúde.

\section{CASUÍSTICA E MÉTODOS}

Estudo transversal realizado com 70 gestantes $(85,4 \%)$ de um total de 82 identificadas no cadastro do Sistema de Monitoramento e Avaliação do
Pré-natal, Parto, Puerpério e Criança de quatro Unidades de Saúde da Família do município de Alfenas-MG, no período de janeiro a junho de 2017. Foram consideradas elegíveis as gestantes de idade superior a vinte anos em qualquer período da gestação que realizavam regularmente do pré-natal e aceitaram, voluntariamente, participar do estudo. Excluíram-se as gestantes consideradas de risco (hipertensas, diabéticas, gestação gemelar) e as que abandonaram o pré-natal.

A coleta de dados foi realizada por meio do atendimento nutricional nas unidades de saúde ou por visitas domiciliares previamente agendadas. $\mathrm{O}$ instrumento utilizado na coleta de dados foi um questionário estruturado com informações socioeconômicas, obstétricas, estilo de vida e nutricionais e a dosagem dos parâmetros hematológicos. A presença de anemia foi identificada por um nível de hemoglobina $<11 \mathrm{~g} / \mathrm{dL}$ obedecendo-se os critérios propostos pela Organização Mundial da Saúde (OMS) ${ }^{9}$.

As medidas antropométricas de peso e altura foram aferidas com base nos critérios de padronização do Ministério da Saúde $(\mathrm{MS})^{10}$. A análise do estado nutricional pré-gestacional e gestacional foram considerados os níveis críticos de Índice de Massa Corporal (IMC) para idade gestacional, proposto por Institute of Medicine (IOM, 2009) e adotados pelo Ministério da Saúde ${ }^{11}$. Para classificar a adequação do ganho de peso gestacional foram utilizados os critérios do Institute of Medicine (IOM, 2009), que estabelece limites para o ganho de peso gestacional de acordo com o estado nutricional inicial da gestante ${ }^{11}$. As gestantes foram classificadas nas seguintes categorias: ganho de peso insuficiente, adequado ou excessivo.

A prática alimentar foi avaliada por meio de questionário de frequência de consumo alimentar, que incluiu alimentos naturais de ferro de origem animal, vegetal e estimuladores de absorção do ferro. Para análise dos dados, os alimentos foram distribuídos em grupos de consumo: frequente, regular e raro. Em seguida, obtiveram-se informações referentes ao consumo alimentar das gestantes também por meio da aplicação do recordatório alimentar de 24 horas (R24h). Para tanto, utilizou-se formulário próprio, auxílio de álbum fotográfico de porções de alimentos e utensílios de cozinha, a fim de se coletar informações referentes 
aos horários das refeições, alimentos, preparações e porções.

O cálculo do recordatório alimentar de 24 horas (R24h) foi realizado com a utilização do software Dietbox ${ }^{\oplus}$. A análise nutricional foi realizada com valores em gramas de macronutrientes e miligramas ou microgramas para micronutrientes, por meio de média e desvio padrão.

Para análise da adequação foram utilizados os valores de referência da Dietary Reference Intakes (DRIs) ${ }^{12-16}$. A probabilidade de adequação para a ingestão dos micronutrientes foi calculada pelo $\mathrm{Z}$ Score $^{17}$, considerando-se como adequada a pontuação igual ou superior a 2 .

$\mathrm{Na}$ análise dos micronutrientes o parâmetro para estimação da adequação de vitaminas e minerais foi a Necessidade Média Estimada (EAR), e para os macronutrientes utilizou-se a Faixa de distribuição aceitável de macronutrientes (AMDR).

Os dados foram analisados considerando que a anemia na gestante foi a variável desfecho e as demais foram as variáveis explanatórias. As variáveis contínuas foram descritas por meio de medidas de tendência central e dispersão e as qualitativas por meio de frequências absolutas e relativas. O teste do Qui-quadrado de Pearson foi utilizado para avaliação das associações entre as variáveis categóricas. As análises estatísticas foram efetuadas utilizando-se o programa Stata, versão 12.0. Para todos os testes utilizados adotou-se o nível de significância de 5\%.
Este estudo foi submetido ao Comitê de Ética em Pesquisa da Universidade Federal de Alfenas- MG, sendo aprovado conforme protocolo $\mathrm{n}^{\circ}$ $1.817 .738 / 2016$. As gestantes que aceitaram participar da pesquisa assinaram o Termo de Consentimento Livre e Esclarecido, sendo garantido o sigilo das informações coletadas e o anonimato do indivíduo, seguindo as Diretrizes e Normas Regulamentadoras de Pesquisa envolvendo seres humanos da Resolução 466/12 do Conselho Nacional de Saúde.

\section{RESULTADOS}

Das 70 gestantes analisadas a maioria encontrava-se na faixa etária de 20 a 40 com a média de idade de $26( \pm 5,95)$ anos. A ocorrência de anemia na amostra foi de $11,43 \%$. Maiores frequências de anemia foram observadas em gestantes entre 31 a 40 anos (12,5\%), que possuíam renda familiar superior a um salário mínimo $(12,7 \%)$, menos de 8 anos de estudo (14,3\%) e solteiras $(15,6 \%)$.Verificou-se maior número de gestantes anêmicas no terceiro trimestre de gestação $(15,0 \%)$, que faziam uso de sulfato ferroso $(11,6 \%)$ e eram tabagistas $(25,0 \%)$. Constatou-se um número relevante de gestantes anêmicas com baixo peso no período pré-gestacional $(14,3 \%)$ e gestacional $(18,0 \%)$ (Tabela 1).

Tabela 1. Anemia em gestantes atendidas na rede pública de saúde, segundo as características demográficas, socioeconômicas, obstétricas e antropométricas. Alfenas-MG, 2017.

\begin{tabular}{|c|c|c|c|c|c|c|}
\hline \multirow{3}{*}{ Variáveis } & \multirow{3}{*}{$\mathbf{N}$} & \multicolumn{4}{|c|}{ Anemia } & \multirow{3}{*}{$\mathbf{p}$} \\
\hline & & \multicolumn{2}{|c|}{ Sim } & \multicolumn{2}{|c|}{ Não } & \\
\hline & & n & $\%$ & $\mathrm{n}$ & $\%$ & \\
\hline Idade & & & & & & 0,878 \\
\hline 20 a 30 anos & 54 & 6 & 11,1 & 48 & 88,9 & \\
\hline 31 a 40 anos & 16 & 2 & 12,5 & 14 & 87,5 & \\
\hline Renda Familiar* & & & & & & 0,291 \\
\hline$<1$ Salário & 7 & 0 & 0 & 7 & 100 & \\
\hline$\geq 1$ Salário & 63 & 8 & 12,7 & 55 & 87,3 & \\
\hline Escolaridade & & & & & & 0,357 \\
\hline$<8$ anos & 42 & 6 & 14,3 & 36 & 85,7 & \\
\hline$\geq 8$ anos & 28 & 2 & 7,1 & 26 & 92,9 & \\
\hline Ocupação & & & & & & 0,601 \\
\hline Do lar & 41 & 4 & 9,8 & 37 & 90,2 & \\
\hline Emprego remunerado & 29 & 4 & 13,8 & 25 & 86,2 & \\
\hline
\end{tabular}




\section{Estado Civil}

Solteira

Casada

Situação de moradia

Própria

Alugada

Trimestre

Primeiro

Segundo

Terceiro

Sulfato ferroso

Sim

Não

Tabagismo

Sim

Não

45

25

20

20

Estado nutricional pré-gestacional
50

23

27

43

27

8

62

$\begin{array}{ll}45 & 7\end{array}$

1

15,6

4,0

10,0

15,0

4,3

14,8

15,0

11,6

11,1

25,0

9,7

6
Baixo peso

Eutrofia

Excesso de peso

7

30

$7 \quad 1$

4

3

Estado nutricional gestacional

$$
\begin{aligned}
& \text { Baixo peso } \\
& \text { Eutrofia }
\end{aligned}
$$

Excesso de peso

Ganho de peso

16
26
28

\begin{abstract}
Insuficiente
Adequado

Excessivo
\end{abstract}

14,3
12,1
10,0

38

24

0,731

0,531

95,7

85,2

85,0

0,947

88,4

88,9

0,200

75,0

90,3

0,677

85,7

87,9

90,0

0,781

$\begin{array}{lll}18,8 & 13 & 81,2\end{array}$

$11,5 \quad 23 \quad 88,5$

$\begin{array}{lll}7,1 & 26 & 92,9\end{array}$

0,830

89,2

82,6

100,0

* Salário mínimo considerado no valor de R\$937,00.

Os resultados obtidos com análise da frequência alimentar são apresentados na Tabela 2. Foi possível observar maior prevalência de anemia nas gestantes que consumiam frequentemente alimentos fonte de ferro como carne vermelha, leguminosas e vegetais verde-escuros entre os nutrimentos facilitadores da absorção do ferro como as frutas cítricas. 
Tabela 2. Anemia em gestantes atendidas na rede pública de saúde, segundo a frequência do consumo alimentar. Alfenas-MG, 2017.

\begin{tabular}{|c|c|c|c|c|c|c|c|c|c|c|c|c|c|}
\hline \multirow{4}{*}{ Alimentos } & \multicolumn{12}{|c|}{ Anemia } & \multirow{3}{*}{$\mathbf{p}$} \\
\hline & \multicolumn{6}{|c|}{ Sim } & \multicolumn{6}{|c|}{ Não } & \\
\hline & \multicolumn{2}{|c|}{ Frequente } & \multicolumn{2}{|c|}{ Regular } & \multicolumn{2}{|c|}{ Raro } & \multicolumn{2}{|c|}{ Frequente } & \multicolumn{2}{|c|}{ Regular } & \multicolumn{2}{|c|}{ Raro } & \\
\hline & $\mathbf{n}$ & $\%$ & $\mathbf{n}$ & $\%$ & $\mathbf{n}$ & $\%$ & $\mathbf{n}$ & $\%$ & $\mathbf{n}$ & $\%$ & $\mathbf{n}$ & $\%$ & \\
\hline Carne vermelha & 7 & 87,5 & 0 & 0 & 1 & 12,5 & 54 & 87,1 & 1 & 1,6 & 7 & 11,3 & 0,933 \\
\hline Fígado/ Vísceras & 1 & 12,5 & 2 & 25,0 & 5 & 62,5 & 7 & 11,3 & 12 & 19,4 & 43 & 69,4 & 0,918 \\
\hline Carne branca & 7 & 87,5 & 0 & 0 & 1 & 12,5 & 52 & 83,8 & 5 & 8,1 & 5 & 8,1 & 0,664 \\
\hline Leguminosa & 7 & 87,5 & 0 & 0 & 1 & 12,5 & 58 & 93,5 & 0 & 0 & 4 & 6,5 & 0,532 \\
\hline Vegetais verde-escuros & 6 & 75,0 & 0 & 0 & 2 & 25,0 & 49 & 79,0 & 3 & 4,8 & 10 & 16,1 & 0,695 \\
\hline Rapadura & 0 & 0 & 0 & 0 & 8 & 100,0 & 5 & 8,1 & 2 & 3,2 & 55 & 88,7 & 0,605 \\
\hline Açúcar mascavo & 0 & 0 & 0 & 0 & 8 & 100,0 & 0 & 0 & 0 & 0 & 62 & 100 & - \\
\hline Frutas. cítricas & 7 & 87,5 & 0 & 0 & 1 & 12,5 & 54 & 87,1 & 2 & 3,2 & 6 & 9,7 & 0,854 \\
\hline Abacate & 0 & 0 & 1 & 12,5 & 7 & 87,5 & 9 & 14,5 & 2 & 3,2 & 51 & 82,3 & 0,272 \\
\hline Sementes/ nozes & 0 & 0 & 1 & 12,5 & 7 & 87,5 & 2 & 3,2 & 0 & 0 & 60 & 96,8 & $0,018^{*}$ \\
\hline Beterraba & 5 & 62,5 & 1 & 12,5 & 2 & 25,0 & 31 & 50,0 & 12 & 19,4 & 19 & 30,6 & 0,791 \\
\hline
\end{tabular}

A Tabela 3 apresenta a ingestão diária de nutrientes e suas respectivas recomendações e avaliações entre as gestantes anêmicas e não anêmicas. Entre os micronutrientes, tanto os minerais ferro, cálcio e zinco, quanto às vitaminas $\mathrm{A}, \mathrm{C}$ e ácido fólico, apresentaram inadequação, em ambos os grupos de gestantes. Nos macronutrientes somente as gestantes não anêmicas apresentaram adequação na ingestão de proteínas no primeiro e terceiro semestre da gestação. A ingestão de carboidratos e lipídeos foi insuficiente em todos os trimestres para ambos os grupos, assim como a ingestão de fibras.

Tabela 3. Anemia em gestantes atendidas na rede pública de saúde, segundo a ingestão diária de nutrientes. Alfenas-MG, 2017.

\begin{tabular}{|c|c|c|c|c|c|c|c|c|c|}
\hline \multirow[t]{3}{*}{ Nutrientes } & \multirow{3}{*}{$\begin{array}{c}\text { EAR/ } \\
\text { AMDR }\end{array}$} & \multicolumn{8}{|c|}{ Anemia } \\
\hline & & \multicolumn{4}{|c|}{ Sim } & \multicolumn{4}{|c|}{ Não } \\
\hline & & $\begin{array}{c}\text { Md } \\
\pm \mathrm{Dp}\end{array}$ & $\begin{array}{l}\text { Mín - } \\
\text { Máx }\end{array}$ & Z-Score & $\begin{array}{c}\text { Adequação } \\
(\%)\end{array}$ & $\begin{array}{c}\text { Md } \\
\pm \mathrm{Dp}\end{array}$ & $\begin{array}{l}\text { Mín- } \\
\text {-Máx }\end{array}$ & $\begin{array}{c}\text { Z-Sco- } \\
\text { re }\end{array}$ & $\begin{array}{c}\text { Adequação } \\
(\%)\end{array}$ \\
\hline Ferro (mg) & 22 & $\begin{array}{r}10,99 \\
\pm 3,61\end{array}$ & $\begin{array}{l}6,34- \\
17,12\end{array}$ & -5 & $\begin{array}{l}\text { Inadequado } \\
(<2)\end{array}$ & $\begin{array}{c}10,79 \\
\pm 4,14\end{array}$ & $\begin{array}{l}3,05- \\
20,33\end{array}$ & $-5,1$ & $\begin{array}{c}\text { Inadequado } \\
(<2)\end{array}$ \\
\hline Cálcio(mg) & 800 & $\begin{array}{c}331,67 \\
\pm \\
254,08\end{array}$ & $\begin{array}{l}32,26- \\
838,34\end{array}$ & $-5,8$ & $\begin{array}{l}\text { Inadequado } \\
\quad(<2)\end{array}$ & $\begin{array}{c}412,64 \\
\pm \\
255,65\end{array}$ & $\begin{array}{l}41,34- \\
1.212,5\end{array}$ & $-4,8$ & $\begin{array}{l}\text { Inadequado } \\
\quad(<2)\end{array}$ \\
\hline Zinco (mg) & 9,5 & $\begin{array}{c}5,96 \\
\pm 2,85\end{array}$ & $\begin{array}{l}2,44- \\
11,83\end{array}$ & $-3,7$ & $\begin{array}{l}\text { Inadequado } \\
\qquad(<2)\end{array}$ & $\begin{array}{r}7,56 \\
\pm 4,9\end{array}$ & $\begin{array}{l}0,82- \\
21,62\end{array}$ & -2 & $\begin{array}{l}\text { Inadequado } \\
\qquad(<2)\end{array}$ \\
\hline Vit. A(mcg) & 550 & $\begin{array}{c}563,03 \\
\pm 555,37\end{array}$ & $\begin{array}{c}35,82- \\
1.431,11\end{array}$ & 0,2 & $\begin{array}{l}\text { Inadequado } \\
\quad(50)\end{array}$ & $\begin{array}{c}595,41 \\
\pm \\
1479,81\end{array}$ & $\begin{array}{l}0,55- \\
20,33\end{array}$ & 0,8 & $\begin{array}{c}\text { Inadequado } \\
(85)\end{array}$ \\
\hline Vit. C(mcg) & 70 & $\begin{array}{r}53,23 \\
\pm 64,5\end{array}$ & $\begin{array}{c}9,62- \\
207,17\end{array}$ & $-2,4$ & $\begin{array}{l}\text { Inadequado } \\
(<2)\end{array}$ & $\begin{array}{c}67,34 \\
\pm 84,84\end{array}$ & $\begin{array}{c}1,31- \\
391,92\end{array}$ & $-0,38$ & $\begin{array}{c}\text { Inadequado } \\
(30)\end{array}$ \\
\hline $\begin{array}{l}\text { Ac. Fólico } \\
\text { (mcg) }\end{array}$ & 520 & $\begin{array}{c}136,9 \\
\pm 82,36\end{array}$ & $\begin{array}{l}51,56- \\
283,78\end{array}$ & $-7,4$ & $\begin{array}{c}\text { Inadequado } \\
(<2)\end{array}$ & $\begin{array}{c}140,9 \\
\pm 77,49\end{array}$ & $\begin{array}{l}43,33- \\
373,03\end{array}$ & $-7,3$ & $\begin{array}{c}\text { Inadequado } \\
(<2)\end{array}$ \\
\hline Fibra (g) & 28 & $\begin{array}{r}15,12 \\
\pm 7,55\end{array}$ & $\begin{array}{l}2,57- \\
26,37\end{array}$ & NA & $\begin{array}{l}\text { Inadequado } \\
\qquad(54)\end{array}$ & $\begin{array}{c}13,63 \\
\pm 6,94\end{array}$ & $1,6-30,58$ & NA & $\begin{array}{c}\text { Inadequado } \\
(48,6)\end{array}$ \\
\hline
\end{tabular}




\begin{tabular}{|c|c|c|c|c|c|c|c|c|c|}
\hline \multicolumn{10}{|l|}{$\begin{array}{c}\text { Primeiro } \\
\text { Trimestre }^{\mathrm{a}}\end{array}$} \\
\hline $\mathrm{CHO}$ (Kcal) & $\begin{array}{l}1.081,35 \text { - } \\
1.561,95^{*}\end{array}$ & 846,68 & 846,68 & NA & Inadequado & $\begin{array}{c}723,82 \\
\pm \\
260,66\end{array}$ & $\begin{array}{l}334,12- \\
1.192,72\end{array}$ & NA & Inadequado \\
\hline PTN (Kcal) & $\begin{array}{l}240,3 \text { - } \\
841,05^{\star}\end{array}$ & 115,56 & 115,56 & NA & Inadequado & $\begin{array}{c}284,65 \\
\pm \\
107,52\end{array}$ & $\begin{array}{c}98,92- \\
517,8\end{array}$ & NA & Adequado \\
\hline LIP(Kcal) & $\begin{array}{l}480,6 \text { - } \\
841,05^{\star}\end{array}$ & 199,35 & 199,35 & NA & Inadequado & $\begin{array}{c}427,39 \\
\pm \\
207,23\end{array}$ & $\begin{array}{c}90,36- \\
854,1\end{array}$ & NA & Inadequado \\
\hline \multicolumn{10}{|l|}{$\begin{array}{l}\text { Segundo } \\
\text { Trimestre }^{\mathrm{b}}\end{array}$} \\
\hline $\mathrm{CHO}$ (Kcal) & $\begin{array}{l}1.234,35 \text { - } \\
1.782,95^{\star}\end{array}$ & $\begin{array}{c}667,99 \\
\pm \\
479,986\end{array}$ & $\begin{array}{c}160,2- \\
1.116,28\end{array}$ & NA & Inadequado & $\begin{array}{c}657,11 \\
\pm \\
305,43\end{array}$ & $\begin{array}{c}114,88- \\
1.196,0\end{array}$ & NA & Inadequado \\
\hline PTN(Kcal) & $\begin{array}{l}274,3- \\
960,05^{*}\end{array}$ & $\begin{array}{c}240,51 \\
\pm 112,17\end{array}$ & $\begin{array}{c}129,36- \\
391,28\end{array}$ & NA & Inadequado & $\begin{array}{c}268,22 \\
\pm \\
135,31\end{array}$ & $\begin{array}{l}26,72- \\
645,24\end{array}$ & NA & Inadequado \\
\hline LIP (Kcal) & $\begin{array}{c}548,6- \\
960,05^{*}\end{array}$ & $\begin{array}{c}381,53 \\
\pm \\
171,74\end{array}$ & $\begin{array}{c}205,29- \\
615,78\end{array}$ & NA & Inadequado & $\begin{array}{c}331,78 \\
\pm \\
179,27\end{array}$ & $\begin{array}{l}63,99- \\
853,92\end{array}$ & NA & Inadequado \\
\hline \multicolumn{10}{|l|}{$\begin{array}{c}\text { Terceiro } \\
\text { Trimestre }^{\mathfrak{c}}\end{array}$} \\
\hline $\mathrm{CHO}$ (Kcal) & $\begin{array}{c}1.284,75- \\
1855,75^{*}\end{array}$ & $\begin{array}{c}616,91 \\
\pm \\
127,68\end{array}$ & $\begin{array}{c}469,56- \\
694,92\end{array}$ & NA & Inadequado & $\begin{array}{c}704,64 \\
\pm \\
309,83\end{array}$ & $\begin{array}{c}341,04- \\
1.419,6\end{array}$ & NA & Inadequado \\
\hline PTN (Kcal) & $\begin{array}{l}285,5- \\
999,25^{*}\end{array}$ & $\begin{array}{l}245,25 \\
\pm 24,36\end{array}$ & $\begin{array}{c}218,16- \\
265,36\end{array}$ & NA & Inadequado & $\begin{array}{c}287,55 \\
\pm \\
145,18\end{array}$ & $\begin{array}{l}47,2- \\
611,44\end{array}$ & NA & Adequado \\
\hline LIP(Kcal) & $\begin{array}{l}571,0- \\
999,25^{\star}\end{array}$ & $\begin{array}{l}365,22 \\
\pm 24,87\end{array}$ & $\begin{array}{c}338,31- \\
387,36\end{array}$ & NA & Inadequado & $\begin{array}{c}436,66 \\
\pm \\
210,14\end{array}$ & $\begin{array}{c}70,02- \\
1.053,72\end{array}$ & NA & Inadequado \\
\hline
\end{tabular}

EAR - Necessidade média estimada; AMDR - Faixas de distribuição aceitável de macronutrientes; Md - Média; Dp - Desvio Padrão; NA - Não aplicável; Carboidrato: CHO; Proteína: PTN; Lipídeo: LIP. *Calculado em função do valor energético total. $1^{\circ}$ Trimestre $=2.403 \mathrm{Kcal},{ }^{\mathrm{a}}=\mathrm{n}: 1 ; 2^{\circ}$ Trimestre $=2.743 \mathrm{Kcal},{ }^{\mathrm{b}}=\mathrm{n}: 4 ; 3^{\circ}$ Trimestre $=2.855 \mathrm{Kcal},{ }^{\mathrm{c}}=\mathrm{n}: 3$. Carboidrato $=45$ a $65 \%$ do valor energético total; Proteína $=10$ a $35 \%$ do valor energético total. Lipídeo $=20$ a 35\% do valor energético total.

\section{DISCUSSÃO}

A ocorrência de anemia na amostra estudada foi de $11,43 \%$, valor este que se enquadra em um percentual inferior ao índice considerado pela OMS como um problema de saúde pública grave (> 40\%), porém dentro da margem para classificação como um problema de saúde pública leve $(5-19,9 \%)^{10}$. Tal ocorrência identificada confirma as disparidades regionais da prevalência da anemia gestacional encontrada em diferentes estudos pontuais conduzidos no Brasil ${ }^{6,7}$. Entretanto, foi supe- rior à frequência de anemia $(4,8 \%)$ encontrada em estudo conduzido com gestantes atendidas em ambulatório de pré-natal da rede pública de Cuiabá-MT, Centro-Oeste do Brasil ${ }^{2}$. A menor prevalência de anemia nesta pesquisa poderia ser justificada, em partes, pelo fato de as gestantes incluídas serem procedentes de área urbana, visto que menores prevalências da patologia têm sido observadas em gestantes da área urbana, quando comparadas com aquelas procedentes da zona rural ${ }^{7,18}$.

Os resultados encontrados quanto às características demográficas e socioeconômicas corroboram com os resultados de autores que apontam 
também maiores ocorrências de anemia nas gestantes com renda entre um e dois salários mínimos e escolaridade inferior nove anos de estudo ${ }^{19}$. Tais indicadores como prevalência de anemia em mulheres solteiras e com baixa escolaridade constituem fatores de risco para desfechos perinatais ruins e repercutem, ainda, negativamente no consumo de alguns alimentos em detrimento de outros e nas condições de vida e saúde, já que a baixa escolaridade pode também levar a maior dificuldade em compreender os cuidados com a saúde da gestante acarretando início tardio do pré-natal ou ausência dele, alimentação e hábitos de vida inadequados ${ }^{20}$. Mas, estudo sugere que a presença paterna favorece o desenvolvimento de uma gestação confortável, por transmitir segurança e divisão e/ou compartilha das dificuldades e das responsabilidades desta fase ${ }^{21}$.

Esta pesquisa mostrou maior ocorrência da anemia conforme a evolução da gestação. Esses achados foram similares aos observados em estudo conduzido com mulheres grávidas no Rio Grande do Sul, que encontraram 17,5\% de gestantes anêmicas ao fim da gestação, observando-se que a prevalência diferenciou conforme o trimestre gestacional $(10,0 \%$ no primeiro, $13,2 \%$ no segundo e $17,5 \%$ no terceiro trimestre $)^{22}$. No presente estudo, chama atenção a presença da anemia em gestantes que faziam uso de suplemento antianêmico, o que sugere que mesmo referindo uso da suplementação, não apresentavam redução da prevalência da patologia. Tal fato chama a atenção e sugere a averiguação real da utilização desse suplemento conforme a prescrição, pois sua efetividade é bem respaldada na literatura ${ }^{23}$.

Os dados encontrados nesta pesquisa quanto ao uso do tabaco evidenciam a necessidade de maior discussão com a comunidade a respeito das implicações do tabagismo durante a gestação para o feto, que é um dos grandes causadores de abortos, ruptura de membrana e deslocamento de placenta ${ }^{24}$.

Estudo sobre fatores associados à anemia em gestantes frisa que o estado nutricional e o ganho de peso materno são fatores importantes para um bom êxito da gestação e que gestantes anêmicas com baixo peso pré-gestacional e gestacional são mais propensas a apresentarem anemia. No entanto esse mesmo estudo encontrou gestantes anêmicas em mulheres abaixo de 20 anos $(12,50 \%)^{[18]}$
A inadequação na ingestão de ferro, cálcio e ácido fólico nas gestantes foi semelhante ao encontrado em dois estudos realizados em São Pau$10^{25,26}$, assim como a inadequação na ingestão de carboidratos em um estudo no Piauí ${ }^{20}$. A inadequação na ingestão de proteínas pode ser explicada devido à baixa adesão no consumo durante a gestação, segundo relatado pelas gestantes. Os achados quando a ingestão alimentar evidencia a importância da orientação nutricional em programas públicos voltados para a saúde da mulher, especialmente às gestantes ${ }^{21,27}$.

Ressalta-se como limitações desse estudo, a impossibilidade de aplicação de mais de um recordatório de 24 horas por gestante estudada, conforme a recomendação para pesquisas populacionais ${ }^{28}$ e o estudo transversal, que impossibilita o estabelecimento de relações causais entre o desfecho e as variáveis exploratórias. Ainda, o tamanho amostral e baixa ocorrência de anemia identificada também podem ter contribuído para associação não estatisticamente significante entre o desfecho "anemia" e variáveis reconhecidas pela literatura contribuintes para o evento.

Os resultados evidenciaram baixa prevalência de anemia em gestantes do município de Alfenas, caracterizando um problema leve de saúde pública. Ainda, constatou-se uma situação importante quanto a inadequação do consumo alimentar, visto que pode desencadear consequências para gestante e o feto, principalmente no grupo encontrado porque são tem idade acima de 30 anos, tabagistas e consequentemente mais predispostas a doenças crônicas e circulatórias.

Contudo, faz-se necessário novas investigações sobre os fatores associados à ocorrência dessa deficiência nutricional, destacando-se a importância da assistência à saúde como fator de prevenção à anemia e demais intercorrências gestacionais, o que permitirá uma assistência adequada na gravidez.

\section{REFERÊNCIAS}

1. Horowitz KM, Ingardia CJ, Borgida AF. Anemia in pregnancy. Clin Lab Med. 2013;33(2):281-91.

2. Camargo RMS, Espinosa MM, Pereira SF, Schirmer J. Prevalência de anemia e deficiência de ferro: relação com índice de massa corporal em gestantes do Centro-Oeste do Brasil. Medicina (Ribeirão Preto). 2013;46(2):118-27. 
3. Araújo CRMA, Uchimura TT, Fujimori E, Nishida FS, Veloso GBL, Szarfarc SC. Níveis de hemoglobina e prevalência de anemia em gestantes atendidas em unidades básicas de saúde, antes e após a fortificação das farinhas com ferro. Rev Bras Epidemiol. 2013;16(2):535-45.

4. Campigotto AC, Farias MCAD, Pinto DCF, Albuquerque FGF. Factors relating to iron deficiency anemia in pregnancy: an integrative review. Int Arch Med. 2015;8(159):1-11.

5. Onoh RC, Ezeonu OP, Umeora OUJ, Onoh TJP, Anozie BO, Onyebuchi AK. Anemia in pregnancy in Abakaliki, South East Nigeria. Int J Gynaecol Obstet. 2014;125(3):280.

6. World Health Organization (WHO). The global prevalence of anaemia in 2011. Geneva: WHO; 2015.

7. Fujimori E, Sato APS, Szarfarc SC, Veiga GV, Oliveira VA, Colli C, et al. Anemia em gestantes brasileiras antes e após a fortificação das farinhas com ferro. Rev Saúde Pública. 2011;45(6):1027-35.

8. Townsley DM. Hematologic complications of pregnancy. Semin Hematol. 2013;50(3):222-31.

9. World Health Organization (WHO). Iron deficiency anaemia: assessment, prevention, and control: a guide for programme managers. Geneva, 2001.

10. Ministério da Saúde. Orientações para coleta e análise de dados antropométricos em serviços de saúde: norma técnica do sistema de Vigilância Alimentar e Nutricional - SISVAN. Brasília, 2011.

11. Institute of Medicine, Food and Nutrition Board. Dietary Reference Intakes for Energy, Carbohydrate, Fiber, Fat, Fatty Acids, Cholesterol, Protein, and Amino Acids (Macronutrients). Washington, DC: National Academies Press; 2005.

12. Institute of Medicine, Food and Nutrition Board. Dietary Reference Intakes for Vitamin A, Vitamin K, Arsenic, Boron, Chromium, Copper, Iodine, Iron, Manganese, Molybdenum, Nickel, Silicon, Vanadium, and Zinc. Washington, DC: National Academies Press; 2000.

13. Institute of Medicine, Food and Nutrition Board. Dietary References Intakes for Calcium and Vitamin D. (uncorrected proofs) Washington, DC: National Academies Press; 2010.

14. Institute of Medicine, Food and Nutrition Board. Dietary Reference Intakes for Thiamin, Riboflavin, Niacin, Vitamin B6, Folate, Vitamin B12, Pantothenic Acid, Biotin, and Choline. Washington, DC: National Academies Press; 1998.

15. Institute of Medicine, Food and Nutrition Board. Dietary Reference Intakes for Vitamin C, Vitamin E, Selenium and Carotenoids. Washington, DC: National Academies Press; 2000.

16. American Dietetic Association. Practice Paper of the American Dietetic Association: Using the Dietary Reference Intakes. J Am Diet Assoc. 2011;111(5):762-70.
17. Oliveira ACM, Barros AMR, Ferreira RC. Fatores de associados à anemia em gestantes da rede pública de saúde de uma capital do Nordeste do Brasil. Rev Bras Ginecol Obst. 2015;37(11):505-11.

18. Paula WKAS, Gomes EAS, Silva IC. Prevalência de anemia em gestantes acompanhadas nas unidades básicas de saúde do município de Caruaru-PE. Demetra. 2016;11(2):415-26.

19. Lacerda KSS, Frota KMG, Freire JAP, Voci SM. Prevalência da inadequação no consumo de nutrientes entre gestantes atendidas em unidades básicas de saúde. Rev Bras Promoç Saúde. 2014;27(3):357-64.

20. Araújo ES, Santana JM, Brito SM, Santos DB. Consumo alimentar de gestantes atendidas em Unidades de Saúde. Mundo da Saúde. 2016;40(1):28-37.

21. Silva DG, Macedo NB. Associação entre ganho de peso gestacional e prognóstico da gestação. Scientia Medica. 2014; 24 (3): 229-236.

22. Dani C, Rossetto S, Castro SM, Wagner SC. Prevalência da anemia e deficiências nutricionais, através de diferentes parâmetros laboratoriais, em mulheres grávidas atendidas em dois serviços de saúde pública no Rio Grande do Sul. RBAC. 2008;40(3):171-75.

23. Ministério da Saúde, Secretaria de Atenção à Saúde. Departamento de Atenção Básica. Programa Nacional de Suplementação de Ferro: manual de condutas gerais. Brasília: Ministério da Saúde; 2013.

24. Renner FW, Costa BP, Figueira FP, Ebert JP, Nascimento LS, Ferrari L, et al. Avaliação do uso de drogas por gestantes atendidas em hospital de ensino do interior do Rio Grande do Sul. R Epidemiol Control Infec. 2016;6(2):68-73.

25. Sato APS, Fujimori E, Szarfarc SC, Borges ALV, Tsunechiro MA. Consumo alimentar e ingestão de ferro de gestantes e mulheres em idade reprodutiva. Rev Latino-Am Enfermagem. 2010;18(2):113-21.

26. Viana JML, Tsunechiro MA, Bonadio I, Fujimori E, Santos AU, Sato APS, et al. Adequação do consumo de ferro por gestantes e mulheres em idade fértil atendidas em um serviço de pré-natal. Mundo da Saúde. 2009;33(3):286-93.

27. Andrade BD, Silva ACP, Santos MTM, Campos T, Luquetti SCPD, Cândido APC, et al. Fatores nutricionais e sociais de importância para o resultado da gestação, em mulheres em acompanhamento na rede de atenção primária de Juiz de Fora. Rev Med Minas Gerais. 2015;25(3):344-52.

28. Fisberg RM, Colucci ACA, Morimoto JM, Marchioni DML. Questionário de frequência alimentar para adultos com base em estudo populacional. Rev Saúde Pública. 2008;42(3):550-54

Como citar este artigo:

Luiz AAO, Santos MB, Brito TRP, Gradim CVC, Lima DB. Anemia em Gestantes Atendidas na Rede Pública. Rev. Aten. Saúde 2019;17(59):73-81. 